\title{
Primary Exploration of Rationality of Industry Distribution and Evaluation Model about Sustainable Development Suitability
}

\author{
Chengzeng $\mathrm{You}^{1,2}$, Ling Peng ${ }^{1, *}$, Tong $\mathrm{Wu}^{1,2}$ and Yuan $\mathrm{Hu}^{1,2}$ \\ ${ }^{1}$ Institute of Remote Sensing and Digital Earth, CAS, Beijing 100094, China \\ ${ }^{2}$ University of Chinese Academy of Sciences, Beijing 100049, China \\ ${ }^{*}$ Corresponding author
}

\begin{abstract}
To tackle the problem of scientific evaluation of local industry development and distribution, we focused on two sub problems, where one was the spatial distribution rationality evaluation of multi-scale industrial clusters, and the other was the sustainable development suitability evaluation of industrial clusters. To solve the first subproblem, based on the "Industrial Cluster Theory" and the original "Industrial Cluster Impact", we adopted spatial analysis model and the methods of data mining to explore the aggregation degree, related influence factors and the interaction impact (between the various influence factors) of industrial clusters. To solve the second subproblem, we combined "Michael Porter Diamond Model", and then studied the principles for the choice of indicators like integrity, technicality and sustainability, forming a set of selection method of evaluation index based on "Industrial Cluster Theory". At last, to evaluate the rationality of industry distribution and sustainable development suitability faster, and get an industrial evaluation model of universality, and lay foundations for the follow-up study, we established a set of industrial clusters' index system of sustainable development suitability.
\end{abstract}

Keywords—rationality; industry distribution; sustainable development suitability; evaluation model

\section{INTRODUCTION}

Industry is the lifeblood of the national economy. Whether an industry has reasonable space distribution and whether an industry can achieve sustained development are the most concerned scientific questions of decision maker of government and enterprises. To solve the first subproblem, based on the clustering theory and industrial elements, we proposed a prototype of industrial cluster impact. Moreover, to analyze the multi-scale and all-round spatial layout rationality of industrial cluster, we researched the influence factors of industrial clusters and the interaction of different influence factors using geospatial analysis models and data mining methods, based on resources, economy, population and other multi-source multi-dimensional data. On this basis, focusing on the second subproblem, we applied the comprehensive evaluation theory of fusion statistics and economic competitive advantage theory to research a set of criterions, analysis methods and evaluation model of industrial sustainable development suitability evaluation, which can provide scientifically evaluation basis and decision support for the reasonable planning of industrial layout and optimization adjustment of industrial structure.

\section{RATIONALITY OF INDUSTRY DISTRIBUTION}

\section{A. Industrial Cluster Theory}

"Industrial Cluster Theory" [1], which was called "new theory of regional economic development[2]", was proposed by Michael Porter who thought that industrial cluster is a group of geographically close interrelated companies and related institutions. They are linked together or related to a specific industry area, because they are connected by generality and complementarity. Based on the clustering theory and industrial elements, rationality of industry distribution was studied.

\section{B. Prototype of Industrial Cluster Impact}

Based on "Industrial Cluster Theory", we proposed a prototype of industrial cluster impact. We believed that every cluster is multi-scale, and a little cluster is contained in a big cluster, and a big cluster is contained in a bigger cluster. Clusters may influence each other because of their own characters (Characters of a cluster are defined by resources surrounding. For example, if one cluster depends on the transportation to develop, we define this cluster as "transportation-dependent". And the characters of a big cluster depends on characters of all its little clusters). Clusters of the same characters will reject each other to compete and clusters of the different characters will attract each other to cooperate. The influence of industrial clusters (of the same level) can be divided into two types: "internal tension" and "external impact", as shown in the figure 1 : 


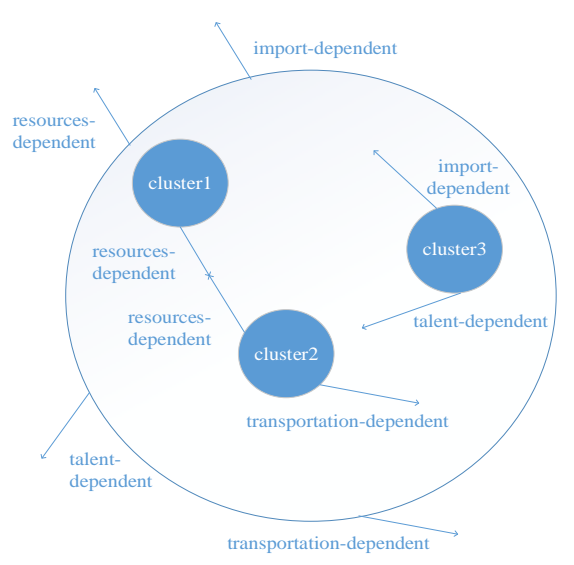

FIGURE I. CONCEPT MODEL OF“INTERNAL TENSION” AND "EXTERNAL IMPACT"

For "internal tension", it happens in one big cluster which include many little clusters. Little clusters may attract or reject each other, and the big cluster will perform its own characters in all after the interaction between the little clusters.

For "external impact", it happens when a cluster 1 which is influenced by many other clusters (all clusters are at the same level) which may attract or reject cluster 1 . All the influence which can be added up is called "external impact" of cluster 1 .

\section{Analysis of Rationality of Industry Distribution}

On the basis of the "Industrial Cluster Theory" and "Industrial Cluster Impact”, we adopted spatial analysis model and the methods of data mining, to explore the aggregation degree, related influence factors and the interaction impact (between the various influence factors) of industrial clusters. And the rationality of industry distribution include three aspects, as shown in the figure 2 :

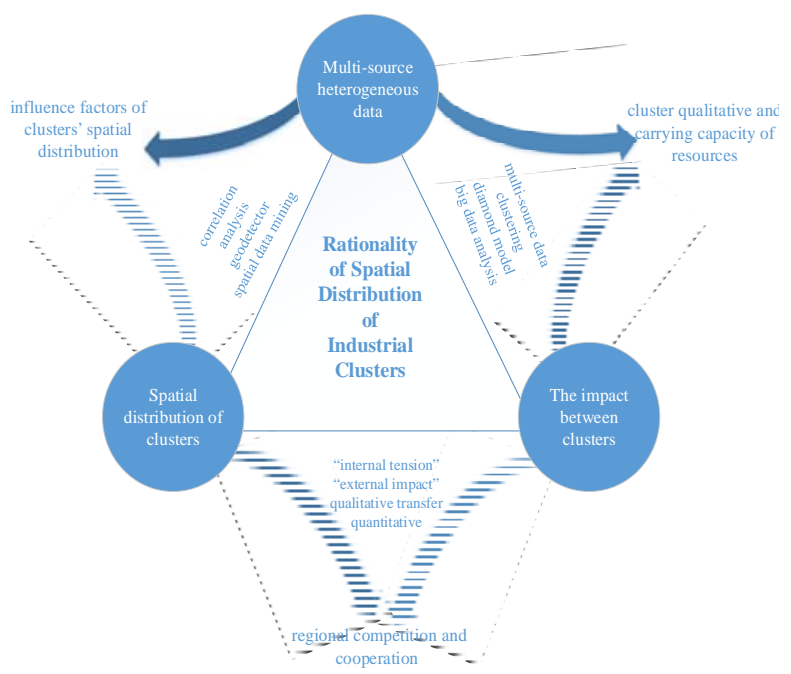

FIGURE II. ANALYSIS MODEL OF RATIONALITY OF INDUSTRY DISTRIBUTION
Multisource heterogeneous data which has spatial property, makes clusters have its own characters. With multisource heterogeneous data and spatial distribution of clusters, we can analyze influence factors of clusters' spatial distribution.

Spatial distribution of clusters was studied by the methods of geographical spatial analysis (such as LISA [3, 4], Moran's I $[5,6]$, Identification of Regional Industry Clusters [7], Coefficient of Variation [8] etc.). And the impact between clusters which is influenced by the cluster's spatial distribution, is stronger while the distance between two clusters is nearer (on the contrary, the impact is lighter while the distance is farther). With clusters' spatial distribution and the impact between clusters, we can analyze the situation of regional competition and cooperation.

The impact between clusters depends on the clusters' characters which depend on the multisource heterogeneous data. With the impact between clusters and multisource heterogeneous data, we can analyze the carrying capacity of resources (when the competition of one region is too fierce for the resources to carry, the impact between clusters will disappear.)

\section{Sustainable DeVElopment SUITABILITY}

For the rationality of sustainable development suitability, we first combined "Michael Porter Diamond Model”, and then studied the principles for the choice of indicators like integrity, technicality and sustainability forming a set of selection method of evaluation index based on "Industrial Cluster Theory". At last, we established a set of industrial clusters' index system of sustainable development suitability.

\section{A. Michael Porter Diamond Model}

Michael Porter proposed Diamond Model (as shown in the figure 3) in his book "The Competitive Advantage of Nations". He believed that whether an industry has competitive advantage mainly depends on the following four conditions:

1) Conditions of production factors.

2) Demand conditions.

3) Related supporting industries.

4) Enterprise strategy, enterprise structure and peer competition.

Besides, Porter still mentioned the effect of opportunity and government ${ }^{[1]}$.

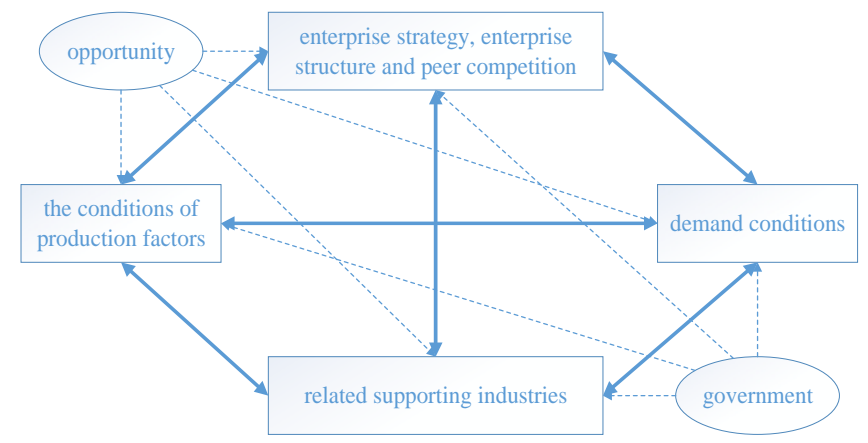

FIGURE III. MICHAEL PORTER DIAMOND MODEL 


\section{B. Principles For the Choice of Indicators}

The choice of indicators must obey some principles to make indicators we chosen more effective, authentic and realistic. We thought there were 7 principles while we were choosing indicators for evaluation:

1) Comprehensiveness: evaluation index system must reflect every aspect of the subject we evaluate.

2) Scientificity: the whole evaluation index system (from indicators to structure, from every indicator's calculation content to methods) must be scientific, reasonable, and accurate.

3) Hierarchy: that is the hierarchical structure of evaluation index system, which can create conditions for factor analysis.

4) Purposiveness: Establishing the evaluation index system must go around evaluation purpose, driving final evaluation conclusion to reflect our true evaluation purpose.

5) Comparability: evaluation index system we establish must be fair for each object we evaluat, so that every object can be comparable.

6) Consistency: different evaluation methods have different requirements for evaluation index system. Sometimes we need to determine methods first and then establish our evaluation index system.

7) Maneuverability: every indicator must be operable, which means accurate data must can be collected timely to be turned into indicators.

\section{Establishing A System of Evaluation}

In this study, establishing an evaluation system about industrial sustainable development suitability was divided into the following steps (as shown in the figure 4):

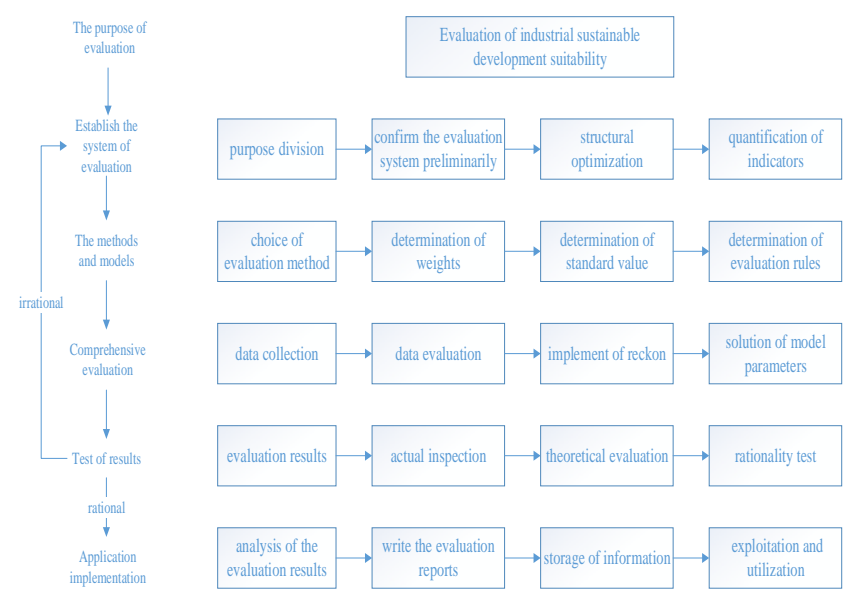

FIGURE IV. FLOW OF ESTABLISHING AN EVALUATION SYSTEM ABOUT INDUSTRIAL SUSTAINABLE DEVELOPMENT SUITABILITY

1) Determining the purpose of evaluation: evaluation system about industrial sustainable development suitability.
2) Establishing the system of evaluation: first, we divided and structured our purpose, so as to confirm the evaluation system preliminarily. And then, we did overall test, individual test and structural optimization of index system. At last, we adopted methods such as fuzzy statistics, classification statistics, expert scoring, qualitative ranking, and scale score and so on to make the qualitative indicators to the appraisal quantitative.

3) Choosing the evaluation methods and models: this part included choosing evaluation method, determination of weights, determining the standard value and evaluation rules.

4) Implement of evaluation: including data collection, data evaluation, data deduction [9] and solving model parameters.

5) Evaluation and test: judging whether the evaluation model, relevant standards, weight and index system are rational or not. If not, we need to return to step 2 .

6) Analysis of evaluation result: including the written analysis of the evaluation results, the writing of evaluation reports, providing and publishing evaluation results, storage of information, exploitation and utilization

\section{Evaluation Model}

To evaluate the rationality of industry distribution and sustainable development suitability faster, and get an industrial evaluation model of universality, and lay foundations for the follow-up study, we built the evaluation model as shown in the figure 5:

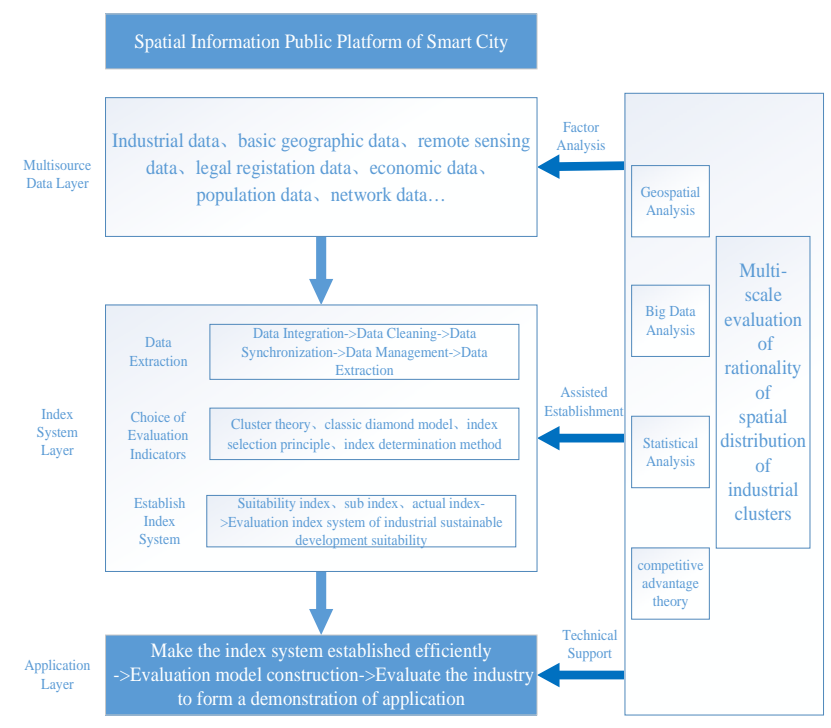

FIGURE V. EVALUATION MODEL OF INDUSTRIAL SUSTAINABLE DEVELOPMENT SUITABILITY

There are three layers in the evaluation model:

a) Multisource Data Layer: depending on the "Spatial Information Public Platform of Smart City", we can easily get plenty of industrial data,basic geographic data, remote sensing data, legal registation data, etc. 
b) Index System Layer: this layer includes data extraction, choice of evaluation indicators and establishment of index system. In data extraction, we need to do data integration, data cleaning, data synchronization, data management and data extraction; In choice of evaluation indicators, we applied "Industrial Cluster Theory”, "Diamond Model”, "index selection principle" and "index determination method" to help the model to choose indicators; In establishment of index system, we fined suitability index, sub index and actual index with levels thus forming evaluation index system of industrial sustainable development suitability.

c) Application Layer: in this layer, we need to make the index system established efficient, and adjust the model according to the practicalities of the application, and evaluate the industry to form a demonstration of application.

In addition, rationality of industry distribution appears in every layer of the model. Actually, it can analyze related factors of industrial sustainable development suitability based on multisource data layer, and help index system layer to establish the evaluation index system of industrial sustainable development suitability, and provide supports for the application layer.

\section{CONCLUSION}

This article made primary exploration of rationality of industry distribution and evaluation model about sustainable development suitability, hoping to build a uniform evaluation standard of industrial sustainable development suitability and provide reference value and guidance significance in analysis theory, technical framework, index system, and evaluation model for the follow-up studies.

\section{ACKNOWLEDGMENT}

This research was sponsored by the National Sciencetechnology Support Plan Project of China (Grant Nos. 2015BAJ02B00)

\section{REFERENCES}

[1] Porter M E. Clusters and New Economics of Competition[J]. 1998, 76(6):77-90

[2] Wei S H, Wang J C, Zhao Y Q. Industrial Cluster:A New Theory of Regional Economic Development[J]. Economic Survey, 2002.

[3] Yang Fan, Du Debin, Duan Dezhong et al. The Intra-metropolitan Location of R\&D-Intensive Manufacturing in Beijing and Shanghai. Scientia Geographica Sinica,2017,37(4):492-501.

[4] Zhang X, Sun L. Manufacture restructuring and main determinants in Beijing metropolitan area[J]. Acta Geographica Sinica, 2012, 67(10):1308-1316.

[5] Gan J, Guo F Y, Chen C, et al. The Spatio-temporal Evolution Characteristics of Urbanization Spatial Differentiation in Northeast China[J]. Scientia Geographica Sinica, 2015, 35(5):565-574.

[6] Troughton E B, Bain C D, Whitesides G M, et al. Monolayer films prepared by the spontaneous self-assembly of symmetrical and unsymmetrical dialkyl sulfides from solution onto gold substrates: structure, properties, and reactivity of constituent functional groups[J]. Langmuir, 1988, 4(2):984-987.

[7] Liu X Q, Sun T S, Guo-Ping L I. Spatial Analysis of Industry Clusters Based on Local Spatial Statistics:A Case Study of Beijing's Manufacturing Industry Clusters[J]. Scientia Geographica Sinica, 2012, 32(5):530-535.
[8] Tian S, Zhang W. Evolution of Spatial Pattern of Chinese Iron and Steel Industry and the Influencing Factors[J]. Progress in Geography, 2009, 28(4):537-545.

[9] Scharstein D, Szeliski R. A Taxonomy and Evaluation of Dense TwoFrame Stereo Correspondence Algorithms[M]. Kluwer Academic Publishers 